\title{
A prospective study of brachytelephalangic chondrodysplasia punctata: identification of arylsulfatase E mutations, functional analysis of novel missense alleles, and determination of potential phenocopies
}

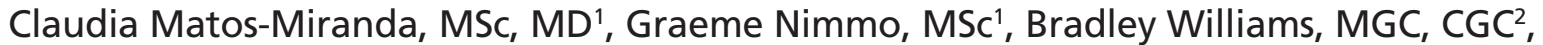 \\ Carolyn Tysoe, PhD 3 , Martina Owens, BS ${ }^{3}$, Sherri Bale, PhD ${ }^{2}$ and Nancy Braverman, MS, MD ${ }^{1,4}$
}

\begin{abstract}
Purpose: The only known genetic cause of brachytelephalangic chondrodysplasia punctata is X-linked chondrodysplasia punctata 1 (CDPX1), which results from a deficiency of arylsulfatase E (ARSE). Historically, ARSE mutations have been identified in only $50 \%$ of male patients, and it was proposed that the remainder might represent phenocopies due to maternal-fetal vitamin $\mathrm{K}$ deficiency and maternal autoimmune diseases.

Methods: To further evaluate causes of brachytelephalangic chondrodysplasia punctata, we established a Collaboration Education and Test Translation program for CDPX1 from 2008 to 2010. Of the 29 male probands identified, 17 had ARSE mutations that included 10 novel missense alleles and one single-codon deletion. To determine pathogenicity of these and additional missense alleles, we transiently expressed them in COS cells and measured arylsulfatase E activity using the artificial substrate, 4-methylumbelliferyl sulfate. In addition, clinical data were collected to investigate maternal effects and genotype-phenotype correlations.
\end{abstract}

Results: In this study, 58\% of males had ARSE mutations. All mutant alleles had negligible arylsulfatase E activity. There were no obvious genotype-phenotype correlations. Maternal etiologies were not reported in most patients.

Conclusion: CDPX1 is caused by loss of arylsulfatase E activity. Around $40 \%$ of male patients with brachytelephalangic chondrodysplasia punctata do not have detectable ARSE mutations or known maternal etiological factors. Improved understanding of arylsulfatase $\mathrm{E}$ function is predicted to illuminate other etiologies for brachytelephalangic chondrodysplasia punctata.

Genet Med 2013:15(8):650-657

Key Words: arylsulfatase E; brachytelephalangic chondrodysplasia punctata; CDPX1; maternal autoimmune disease; vitamin $\mathrm{K}$

stature. Another commonly observed characteristic is mixed conductive and sensorineural hearing loss. Most children have normal intellect and life span, but comorbidities can be present, including compression of the cervical spinal cord associated with cervical vertebral abnormalities or stenosis of the upper and lower airways that result from extensive calcifications within the tracheal and bronchial cartilage. ${ }^{3,4}$ Intrafamilial differences in disease severity have also been documented. ${ }^{3}$

The only known genetic cause of CDPX1 is defects in arylsulfatase E (ARSE). The ARSE gene is located at Xp22.3, within a cluster of contiguous arylsulfatase genes that share high sequence homology, escape $\mathrm{X}$ inactivation, and have pseudogenes on the Y chromosome..$^{5}$ There are 17 sulfatases in the human genome; all share extensive sequence homology and contain a highly conserved cysteine that undergoes a unique posttranslational modification essential for their catalytic activity. ${ }^{6-8}$ ARSE is localized to the Golgi membranes, ${ }^{9}$ and its transcript has been identified in multiple tissues. ${ }^{5}$ Its protein product is a 589 -amino-acid and $60 \mathrm{kD}$ precursor, which is subject

\footnotetext{
${ }^{1}$ Department of Human Genetics, Montreal Children's Hospital Research Institute, McGill University, Montreal, Quebec, Canada; ${ }^{2} \mathrm{GeneDx}$, Gaithersburg, Maryland, USA; ${ }^{3}$ Royal Devon and Exeter Foundation Trust, Exeter, UK; ${ }^{4}$ Department of Pediatrics, Montreal Children's Hospital Research Institute, McGill University, Montreal, Quebec, Canada. Correspondence: Nancy Braverman (nancy.braverman@mcgill.ca)
} 
to $\mathrm{N}$-glycosylation at four potential sites (asparagine residues $58,125,258$, and 344 ) to give a mature $68 \mathrm{kD}$ form. Aside from the conserved active site domain, there are two predicted transmembrane domains, through which it is likely to be anchored to the Golgi cisternae. Its physiological substrate is unknown. Enzymatic assays to determine endogenous ARSE activity in fibroblasts have not been successful thus far because of interference with other highly homologous arylsulfatases. ${ }^{9}$ The ability of ARSE to hydrolyze the fluorogenic 4-methylumbelliferyl (4MU) sulfate permits the use of this artificial substrate to measure its activity in an in vitro system. ${ }^{5}$ Studies showed that the enzyme activity is optimum at neutral $\mathrm{pH}^{5,9}$

By 2008, more than 140 male patients with a CDPX1 phenotype had been reported, and of these, around one-fourth were associated with $\mathrm{Xp}$ deletions or chromosomal rearrangements. Only 67 male probands had undergone ARSE mutation analysis, with mutations identified in $31 .^{3}$ Functional analysis was performed in 8 of 15 reported missense alleles (Supplementary Table S1 online) by expressing the mutant ARSE complementary DNA in mammalian COS1 cells and measuring ARSE activity using $4 \mathrm{MU}$ sulfate. ${ }^{5}$ In these experiments, all missense alleles had negligible activity. ${ }^{9,10}$

Considering the phenotypic resemblance of warfarin embryopathy in early gestation to BCP, Franco et al. ${ }^{5}$ (1995) demonstrated that ARSE activity is inhibited in the presence of warfarin, an anticoagulant drug that decreases amounts of active vitamin $\mathrm{K}$. These findings suggested that cases of BCP without ARSE mutations could be caused by inhibition of a normal ARSE enzyme in fetal development by reducing levels of vitamin $\mathrm{K}$. Because placental transport of vitamin $\mathrm{K}$ is normally reduced, ${ }^{11}$ vitamin $\mathrm{K}$ deficiency in the mother would be expected to result in greater vitamin $\mathrm{K}$ deficiency in the fetus. This hypothesis is attractive given that there have been several reports of BCP in offspring of both sexes in which gestational vitamin $\mathrm{K}$ deficiency was suspected, including mothers with severe hyperemesis gravidarum, ${ }^{12}$ small-intestinal obstruction, ${ }^{13}$ small-bowel syndrome, ${ }^{14}$ pancreatitis, ${ }^{15}$ or biliary lithiasis. ${ }^{16}$ In those cases in which ARSE mutations were evaluated in their affected offspring, none were found. ${ }^{3,13}$ Another group of children with BCP are born to mothers with autoimmune diseases. ${ }^{17-19}$ It was proposed that maternal autoantibodies might perturb fetal vitamin
$\mathrm{K}$ metabolism, ${ }^{20}$ thereby reducing the etiology of all phenocopies to disturbances in vitamin $\mathrm{K}$ metabolism at a critical fetal time period. Although this hypothesis remains to be proven, it was not clear how many patients with BCP might be phenocopies due to maternal vitamin $\mathrm{K}$ deficiency states.

In 2008, through the Collaboration Education and Test Translation program (CETT), ${ }^{21}$ sponsored by the National Institutes of Health Office of Rare Diseases Research, we established a pilot project to identify patients with CDPX1 and its phenocopies. The collaborative group included the clinical laboratory, which performed ARSE gene sequencing in patients referred with CDPX1 phenotypes and collected clinical information from the referring physician using a clinical data sheet designed for the CETT program for CDPX1 (Supplementary Table S2 online). The research laboratory reviewed the clinical data sheets to evaluate genotype-phenotype correlations and performed functional analysis of the novel missense alleles identified. Because the majority of ARSE mutations identified thus far have been unique, functional analysis was important to demonstrate pathogenicity. This was also a unique opportunity to prospectively determine the incidence of CDPX1 phenocopies.

\section{CDPX1 phenotype}

\section{MATERIALS AND METHODS}

CDPX1 phenotype was defined as male sex, nasomaxillary hypoplasia, brachytelephalangy, and punctate calcifications on $\mathrm{X}$-ray in children younger than 3 years.

\section{Clinical data collection}

The data sheet was designed to sample all the major features described in patients with BCP reported in the literature since 1978. In addition, it contained broad questions to identify other etiologies, including maternal vitamin $\mathrm{K}$ deficiency and autoimmune disease, that have been associated with BCP. The final data sheet was approved by the program staff and review board of the CETT program. Informed consent was obtained for the collection of clinical data, along with the consent for ARSE gene sequencing, according to the CETT program requirements. Referring physicians were asked to complete this form (shown in Supplementary Table S2 online) at the time of sample submission. The clinical data sheets were deidentified and then

Table 1 Breakdown of patients and data sheets collected in this project

\begin{tabular}{|c|c|c|c|c|c|}
\hline & & $\begin{array}{c}\text { NIH-CETT program } \\
(2008-2010)\end{array}$ & $\begin{array}{c}\text { Clinical data sheets } \\
\text { obtained }\end{array}$ & $\begin{array}{l}\text { Other referrals } \\
(2008-2010)\end{array}$ & $\begin{array}{c}\text { Clinical data sheets } \\
\text { obtained }\end{array}$ \\
\hline \multirow[t]{3}{*}{ Male } & ARSE mutation identified-CDPX1 & 17 & 13 & 3 & 2 \\
\hline & No ARSE mutation identified & 12 & 6 & & \\
\hline & Brother with CDPX1 & 2 & & 1 & \\
\hline \multirow[t]{4}{*}{ Female } & No ARSE mutation identified & 5 & & & \\
\hline & Carrier mother & 6 & & 3 & \\
\hline & Carrier relative & 2 & & & \\
\hline & Noncarrier relative & 1 & & & \\
\hline
\end{tabular}

ARSE, arylsulfatase E; NIH CETT, National Institutes of Health Collaboration Education and Test Translation. 
sent to the research laboratory for review. See Table 1 for the breakdown of patients involved in this study.

\section{Literature review}

We searched PubMed from 1995 to 2010 to identify ARSE mutations in which functional analysis had not been done. Search terms used were CDPX1, ARSE, X-linked recessive $\mathrm{CDP}$, and BCP.

\section{Molecular analysis}

All 11 exons and intronic flanking sequences of the ARSE gene were Sanger sequenced bidirectionally. In females, targeted array comparative genomic hybridization with exon-level resolution was performed concurrently with sequencing. Targeted mutation analysis was performed in affected male relatives of probands with ARSE mutations and in mothers and other female relatives to evaluate carrier status.

\section{Mutant complementary DNA constructs}

Wild-type ARSE complementary DNA (wtARSE) was engineered in the mammalian expression vector, pALTER-MAX (Promega, Madison, WI) by inserting ARSE exon 2, obtained by RT-PCR from a liver RNA library, into the ARSE complementary DNA clone (GenBank: AA887688.1). It contained

Table 2 ARSE mutations detected in this study

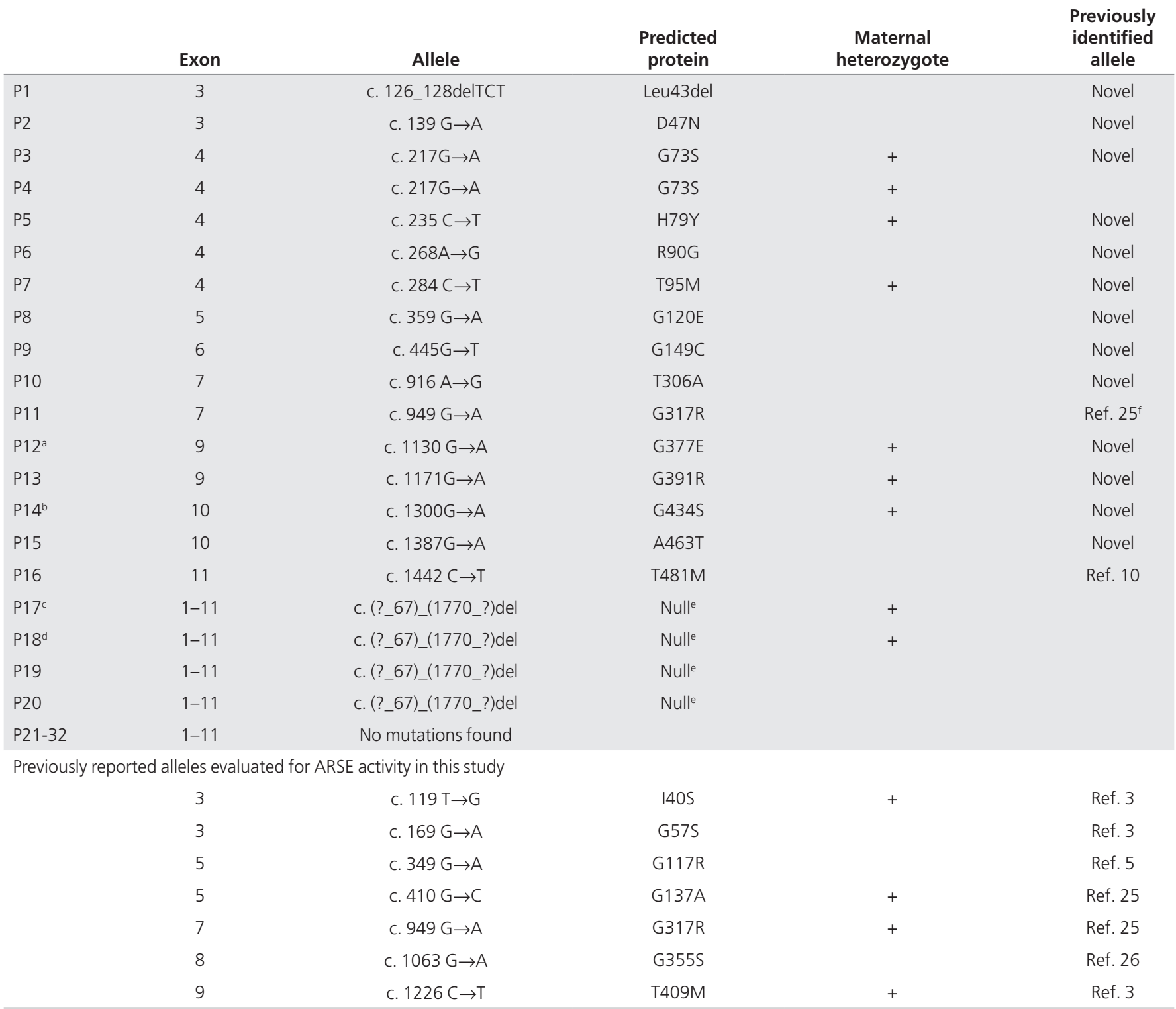

ARSE, arylsulfatase $\mathrm{E}$.

aThis patient has a brother with similar features who was also found to have c. $1130 \mathrm{G} \rightarrow \mathrm{A}$. ${ }^{\mathrm{b}}$ This patient has a brother with similar features who was also found to have $\mathrm{c}$.

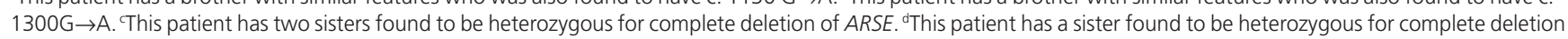
of $A R S E$. ${ }^{e}$ Complete deletions of ARSE are reported in CDPX1; because the break points have not been determined, it is not known if these are identical. ${ }^{\text {TThis }}$ mutation was identified by Sheffield et al. (ref. 25) but there is no functional analysis information on it, indicated by a "?". 

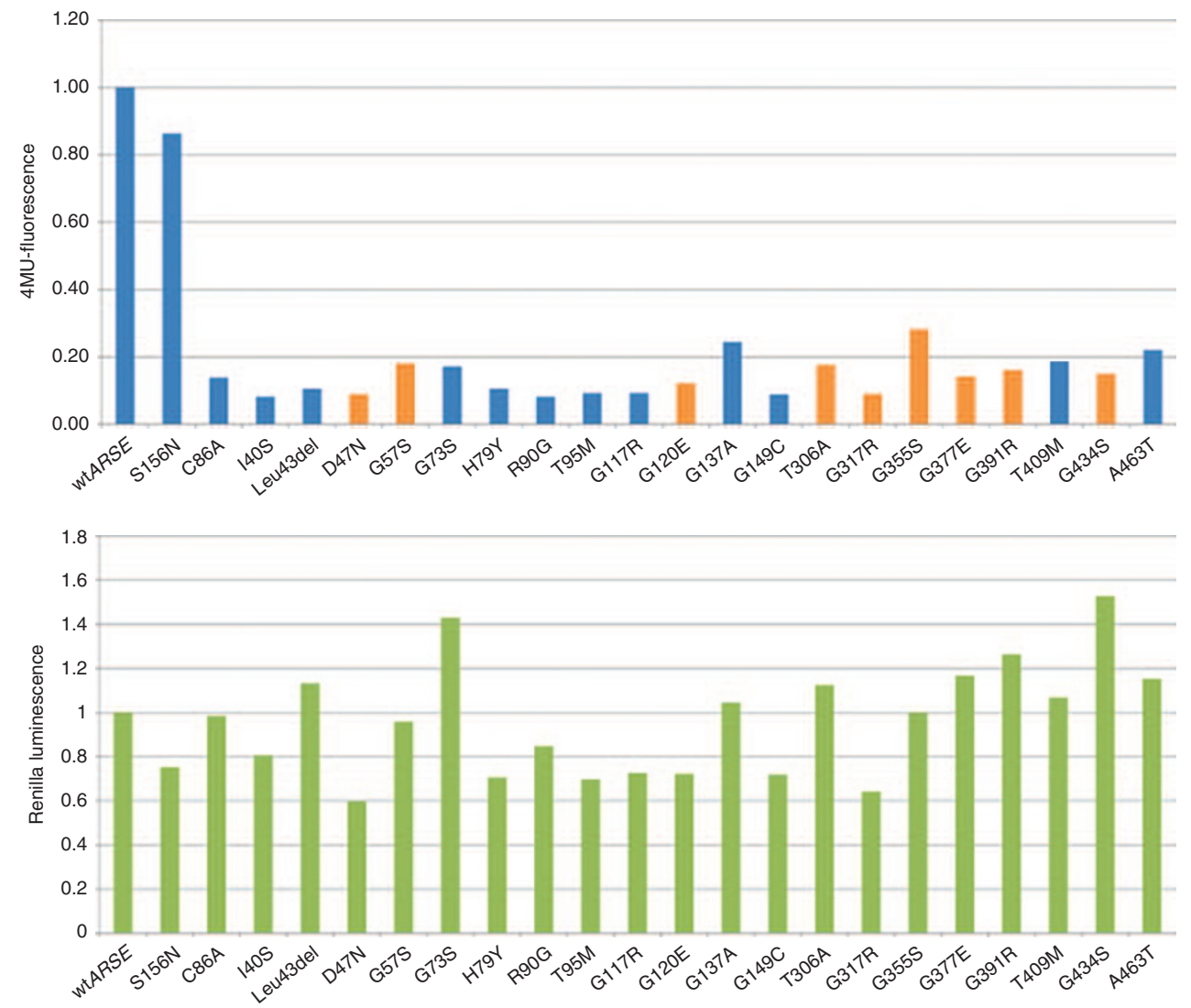

Figure 1 Activity of ARSE missense proteins. Top, mutations are listed on the $X$ axis from $N$ to $C$ terminus of ARSE. C86A alters the putative active site of ARSE and was used as a negative control. S156N is a reported ARSE polymorphism. Missense proteins in orange were subsequently tested for residual activity. The $Y$ axis represents the ratio of averaged fluorescence of the mutant proteins to WTARSE. Bottom, expression of Renilla plasmid cotransfected with each ARSE mutant protein. Y axis shows measured Renilla luminescence units. Experiments represent three independent replications. ARSE, arylsulfatase E; wtARSE, wild-type ARSE.

the open reading frame, bps 1-1856, including 86 nucleotides of the $3^{\prime}$ untranslated region. wtARSE diverges from the reference sequence (NM_000047.2) by having the synonymous substitutions c.4T $\rightarrow \mathrm{C}$ and c.1771 $\mathrm{A} \rightarrow \mathrm{G}$, and the polymorphisms reported in dbSNP: c.495 T $\rightarrow$ C, c.1270 G $\rightarrow$ A, and c.1692 C $\rightarrow \mathrm{T}$. Site-directed mutagenesis of wtARSE was performed using the QuikChange II Site-Directed Mutagenesis Kit (Stratagene, La Jolla, CA) and resequenced after mutagenesis for confirmation. Primers used to generate ARSE mutations are listed in Supplementary Table S3 online.

\section{COS cell transfections}

COS1 cells were maintained in Dulbecco's Modified Eagle Medium supplemented with $10 \%$ fetal bovine serum at $37^{\circ} \mathrm{C}$ and $5 \% \mathrm{CO}_{2}$. Overnight cultures were seeded at $10 \times 10^{5}$ cells per $21 \mathrm{~cm}^{2}$ culture plate and cotransfected with $5 \mu \mathrm{g}$ of ARSE and 2.5 $\mu \mathrm{g}$ of Renilla reniformis luciferase (Promega) plasmids using 15 $\mu \mathrm{l}$ of FuGENE HD (Roche Diagnostics, Mannheim, Germany) according to manufacturer's instructions. Transfections were performed in triplicate. Cells were harvested $48 \mathrm{~h}$ after transfection, washed with phosphate-buffered saline, trypsinized, and resuspended in Dulbecco's Modified Eagle Medium with $10 \%$ fetal bovine serum, centrifuged at $1,000 \mathrm{rpm}$ for $4 \mathrm{~min}$ and resuspended in $200 \mu \mathrm{l}$ of lysis buffer: $0.1 \mathrm{mmol} / \mathrm{l} \mathrm{Tris} \mathrm{HCl}$, $0.15 \mathrm{~mol} / \mathrm{l} \mathrm{NaCl}$, and $1 \%$ Triton X-100 at $\mathrm{pH} 7.5$. The lysate was incubated on ice for $20 \mathrm{~min}$, then centrifuged at 20,800 $\mathrm{rcf} \times$ $20 \mathrm{~min}$ at $4{ }^{\circ} \mathrm{C}$. Supernatant was collected and protein concentration was measured by Bradford assay. For time course experiments, $0.5 \mu \mathrm{g}$ of Renilla plasmid was used and lysis buffer was $0.05 \mathrm{~mol} / \mathrm{l}$ Tris HCI at pH 7.5.

\section{ARSE assay}

The reported assay ${ }^{5,12}$ was modified as follows: incubation mixture $(100 \mu \mathrm{l})$ contained $20 \mu \mathrm{g}$ of lysate and $0.2 \mathrm{mmol} / \mathrm{l} 4 \mathrm{MU}$ sulfate (Sigma-Aldrich, Saint Louis, MO) in $0.05 \mathrm{~mol} / \mathrm{l}$ Tris $\mathrm{HCI}$ buffer ( $\mathrm{pH}$ 7.5). Incubation was performed for $3 \mathrm{~h}$ at 37 ${ }^{\circ} \mathrm{C}$ in a 96-well plate; the reaction was stopped with $1.8 \mathrm{ml}$ of glycine-carbonate buffer ( $\mathrm{pH}$ 10.7). Fluorescence was determined (Wallac1420 Multilabel Counter, software version 3.00; PerkinElmer, Waltham, MA) at $365 \mathrm{~nm}$ (excitation) and $460 \mathrm{~nm}$ (emission). Each sample was run in triplicate and the experiment was repeated three times. Time course experiments on selected mutations were stopped at $0 \mathrm{~h}, 30 \mathrm{~min}, 1 \mathrm{~h}, 2 \mathrm{~h}$, and $2 \mathrm{~h} 30 \mathrm{~min}$. Renilla luminescence was measured by the DualLuciferase Assay and a GloMax 96 Microplate Luminometer (Promega). 
a

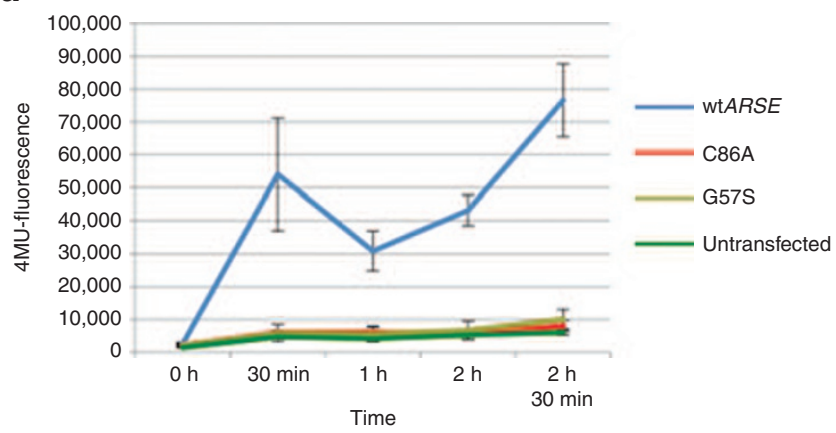

C

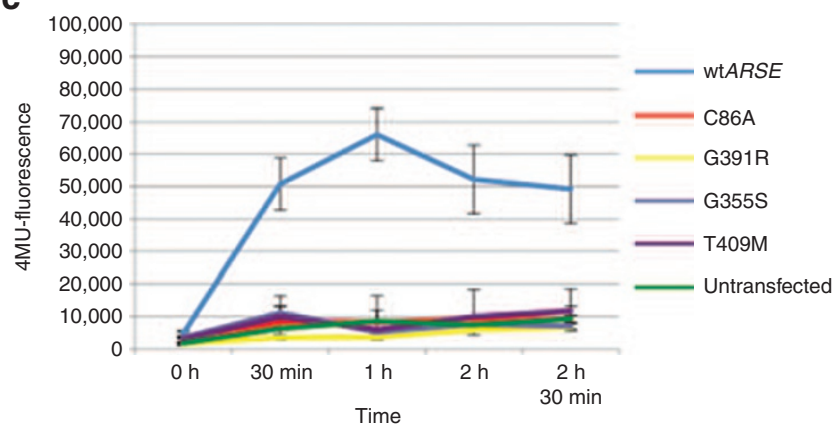

b

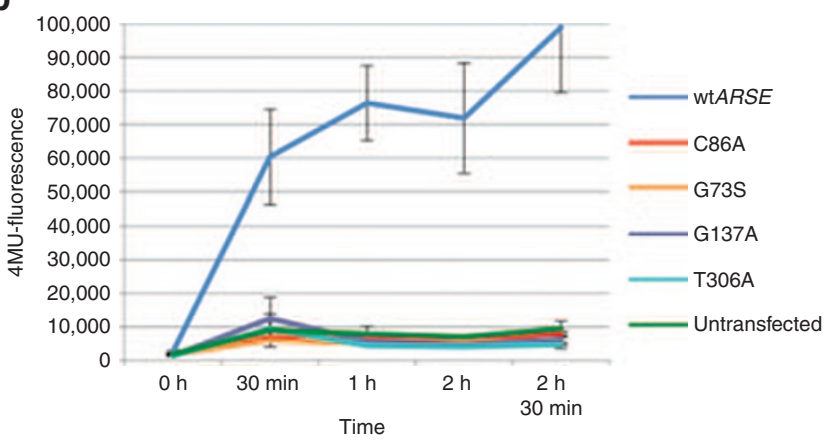

d

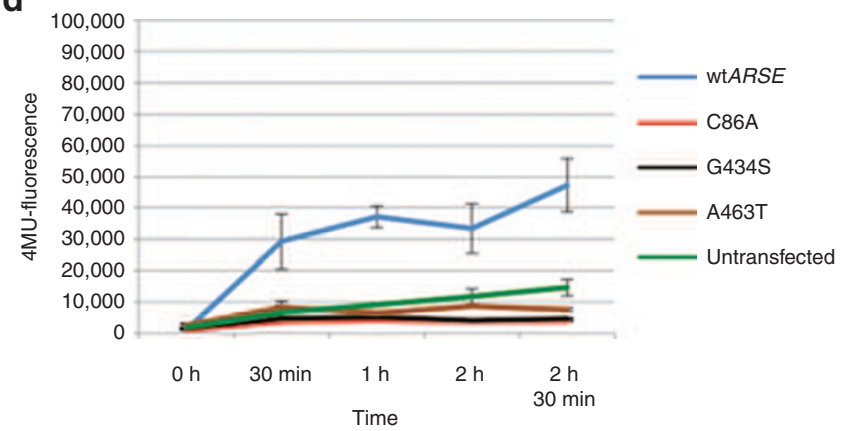

Figure 2 Time course experiments for selected alleles. (a) G57S; (b) G73S, G137A, T306A; (c) G391R, G355S, T409M; (d) G434S and A463T show negligible activity over time compared with wtARSE. The $Y$ axis represents 4-methylumbelliferyl-fluorescence (1.0s) (counts). Error bars represent the SD of the measurements. Experiments represent three independent replications. ARSE, arylsulfatase E; wTARSE, wild-type ARSE.

\section{RESULTS}

\section{Clinical and molecular analysis}

Twenty nine male probands were referred prospectively through the CETT program, and clinical data sheets were collected on 19. Three additional male probands with ARSE missense alleles were contributed by the Molecular Genetics Laboratory of the Royal Devon and Exeter National Health Service Foundation Trust, and clinical data sheets were collected on two of them. From these 21 data sheets, the frequency of relevant clinical features in ARSE mutation-positive and mutation-negative probands was collated in Supplementary Table S4a,b online, respectively.

ARSE mutations were identified in 17/29 male probands from the CETT project and included four complete gene deletions, 1 single-codon deletion, and 12 missense mutations. The mutations identified are shown in Table 2. All missense alleles were predicted to be damaging by either PolyPhen ${ }^{22}$ or $\mathrm{SIFT}^{23}$ analysis, except for T306A, which was predicted to be benign. None of these mutations were polymorphisms identified in dbSNP (V132). ${ }^{24}$ All residues affected by the mutations were evolutionarily conserved among all ARSE proteins and human arylsulfatate C (ARSC).

In addition, four females with clinical features of $\mathrm{BCP}$ were referred for ARSE mutation analysis. No mutations were found, and clinical data were obtained in only one patient. She had nasomaxillary hypoplasia, flattened nose, anteverted nostrils, brachytelephalangy, ichthyosis, delayed motor, and cognitive development. At the age of 11 months, there was no evidence of chondrodysplasia punctata, and her karyotype was normal.
The distribution of all patients included in this project is shown in Table 1.

\section{ARSE functional analysis}

We performed functional analysis on the 12 novel missense alleles, the single codon deletion identified in our cohort, and 7 novel missense alleles reported previously in which functional analysis had not been done $e^{3,5,25,26}$ (Table 2). These 20 $A R S E$ alleles were engineered by site-directed mutagenesis and coexpressed in COS1 cells with Renilla luciferase to control for transfection efficiency. Whole-cell lysates were incubated with $4 \mathrm{MU}$ sulfate for $2 \mathrm{~h}$, and ARSE activity was measured by detection of $4 \mathrm{MU}$ product. We found that all the mutant alleles had negligible ARSE activity, suggesting that they were pathological (Figure 1). However, we observed minimally increased fluorescence in some alleles, especially those located in the C-terminal region, suggesting potential residual activity. To determine if residual activity was present, time course experiments were performed for nine selected alleles. We found that none of these alleles showed an increase of ARSE activity over time as compared with wtARSE (Figure 2a-d).

\section{Spectrum of ARSE mutations}

\section{DISCUSSION}

Twenty nine male probands with CDPX1 phenotype were referred through the CETT program. Molecular analysis identified ARSE mutations in 17, providing a mutation identification rate $\sim 58 \%$, similar to previous reports. ${ }^{3,5,10}$ 


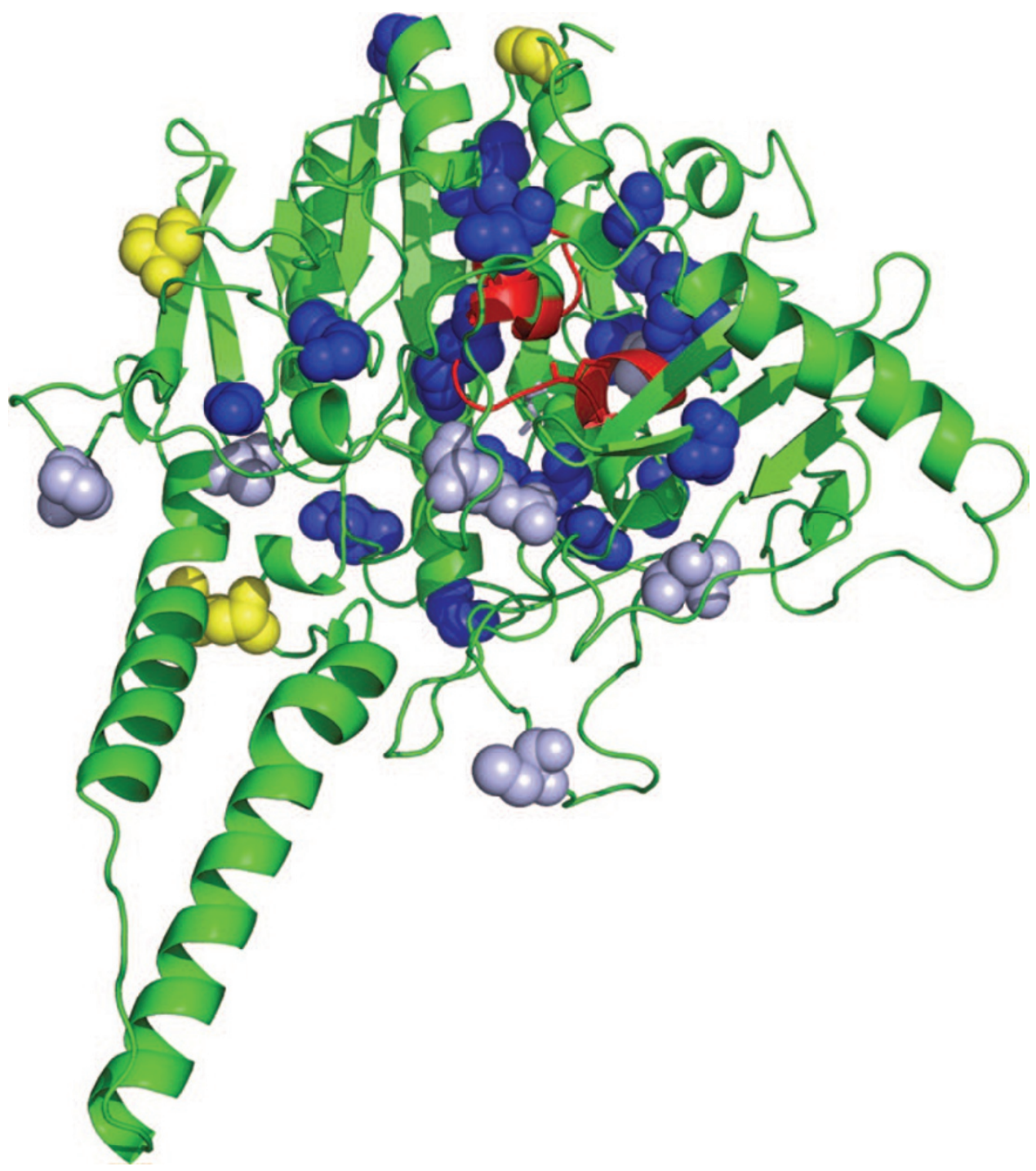

Figure 3 Model of ARSE. ARSE was superimposed on arylsulfatase $C$ crystal structure and visualized by PyMOL. $\beta$ Sheets, connecting loops, and $\alpha$ helices are green. The 18-amino-acid active site is red. ARSE residues involved in mutations are depicted as blue spheres, and polymorphisms are yellow spheres (see Table 1). ARSC mutation residues analyzed by Ghosh (2004) (ref. 28) are light blue spheres. The two transmembrane domains are shown as parallel $\alpha$ helices at the bottom of the molecule. ARSC, arylsulfatate C; ARSE, arylsulfatase E.

Although the majority of ARSE mutations identified overall have been unique, several reported ARSE mutations occurred more than once in unrelated families, including G73S, G137A, G317A, T481M, P578S, and W581X. Of these, only G73S and T481M represent transitions at CpG dinucleotides. We also noted that the maternal carrier frequency may be high. Although only nine mothers were tested in this study, all were found to be carriers of the ARSE mutations identified in their sons. Similarly, nine mothers of previously reported males with CDPX1 who had carrier testing were found to be carriers. ${ }^{3,25,27}$ Maternal carriers were identified among both ARSE deletion and point mutation alleles (see Table 2). We suggest that the high frequency of maternal carriers, if proven, could be secondary to de novo mutations occurring more often in the germline of the maternal grandfather. Alternatively, given that patients with
CDPX1 often survive and reproduce, the frequency of new mutations may be low.

\section{Absence of genotype-phenotype association}

The majority of missense substitutions were located in residues that were conserved in evolution for both ARSE and other arylsulfatases, ASA, ASB, ASC, ASD, ASH, and ASF. All missense alleles were distributed along most of the full protein and had negligible activity as compared with wtARSE. Of note, there were no missense alleles identified in exon 2 , which contains the start methionine codon, and none in the putative transmembrane domains. These results, taken together with indistinguishable phenotypes of patients with ARSE gene deletions and nonsense and missense alleles, indicate the absence of an association between mutation and disease severity. This is further supported by variation in 
phenotype severity between affected males within the same family. ${ }^{3}$

It is possible that the missense alleles interfere with the proper localization of the protein or result in a catalytically inactive or misfolded and degraded protein. Daniele et al. ${ }^{9}$ showed that the ARSE missense alleles, R11P, G137V, G245R, and G492Y, when overexpressed in COS cells had negligible activity but retained stability and Golgi location, suggesting that these residues may be important for the catalytic activity of ARSE.

\section{ARSE protein modeling}

ARSE is most closely related evolutionarily to ARSC and likely derives from this gene by duplication. ${ }^{6}$ The amino acid identity between ARSE and ARSC is 51\%. Recently, the crystal structure of ARSC, a transmembrane endoplasmic reticulum protein, was determined. ${ }^{28}$ The crystal structure implies that the active site rests near the membrane surface, suggesting an intriguing role for the lipid bilayer in catalysis. Sequence analysis predicts that ARSE also contains two putative membrane-spanning domains, which likely anchor ARSE to Golgi membranes. ${ }^{9}$ To further evaluate the effect of ARSE missense alleles on protein function, we modeled ARSE onto ARSC ${ }^{29}$ using the automated alignment mode in SWISS-MODEL. ${ }^{30}$ Structural 3D assessment for this model was created using the PyMOL Molecular Graphics System (Version 1.2r3pre; Schrödinger, LLC). Using this model, we visualized the location of the ARSE missense alleles identified in this article, as well as three known polymorphisms (Figure 3). Of note, we found that most mutations clustered around the active site (shown in red in Figure 3), similar to previously evaluated ARSC missense mutations suspected of impairing catalytic activity. ${ }^{28} \mathrm{~A}$ smaller group of three mutations clustered around an ARSC mutation, also known to be inactive, that was positioned in a loop that might associate with the membrane (Figure 3). Of note, the known polymorphisms were all positioned toward the external surface. As previously predicted, there were no mutations located in the transmembrane domains. This structural comparison suggests that ARSE missense mutations inactivate ARSE by impairing its catalytic site.

\section{Clinical comparison of BCP patients with and without ARSE mutations}

Clinical data sheets were returned on 21/32 male probands (including those from the United Kingdom) but were not always fully completed. Nevertheless, the frequency of clinical findings (Supplementary Table S4a,b online) was similar to those reported before. ${ }^{3}$ In exception, rhizomelia and ichthyosis, atypical findings for CDPX1, were reported once in patients with ARSE mutations R90G and D47N, respectively. Ganglioneuroblastoma was reported once in a patient without an ARSE mutation. Cervical spine abnormalities, frequently reported in patients with $\mathrm{BCP}$, were not reported in the group without ARSE mutations in this study. Most surprising was the absence of information related to potential phenocopies in the ARSE mutation-negative group. The limitations of this data collection included its nonmandatory requirement to be completed. In addition, more specific questions on maternal vitamin $\mathrm{K}$ deficiency states should have been included. These features limit the interpretation of the data.

\section{Etiologies for BCP in patients without ARSE mutations}

From the 12 male probands identified without ARSE mutations, there were no mothers reported with conditions previously associated with gestational vitamin $\mathrm{K}$ deficiency. With the caveat that the clinical data sheets might be incomplete for maternal history, it seems likely that genetic heterogeneity or other environmental factors not considered also contribute to mutation-negative patients. Future studies could evaluate the X-linked ARSE paralogs ARSD, ARSF, and ARSH. ARSD has been sequenced in some patients with BCP previously without mutations reported. ${ }^{5,25}$ Improved understanding of ARSE function would help to identify additional pathway proteins that, when defective, could be candidates for causing BCP.

One of these pathways may involve vitamin $\mathrm{K}$, as fetal vitamin $\mathrm{K}$ deficiency in early gestation may be responsible for an unknown proportion of BCP phenotypes. ${ }^{3,12,13,16}$ Supporting these observations, offspring of pregnant women receiving warfarin showed shortening and flattening of the nose, epiphyseal stippling, and cerebral hemorrhage. ${ }^{31}$ Warfarin inhibits the VKORC1 complex, which recycles vitamin K. Offspring of pregnant rats treated with warfarin showed calcification and disruption of hypertrophic chondrocytes in long-bone epiphyses. These studies indicate that early gestational warfarin exposure results in bone dysplasia, whereas later exposure affects coagulation factors. Reduced bone $\gamma$-carboxyglutamate levels in the pups suggested that this phenotype was related to inhibited synthesis of vitamin K-dependent skeletal proteins. ${ }^{32}$ Vitamin $\mathrm{K}$-dependent proteins are activated in the endoplasmic reticulum through posttranslational modification by $\gamma$-glutamyl carboxylase. $\gamma$-Glutamyl carboxylase adds $\mathrm{CO}_{2}$ to a bound glutamate residue to form $\gamma$-carboxyglutamate. Vitamin $\mathrm{K}$ is a cofactor in this reaction and is simultaneously oxidized. ${ }^{33,34} \gamma$-Carboxyglutamate or Gla residues bind calcium with high affinity. Vitamin $\mathrm{K}$-dependent proteins involved in bone metabolism are bone Gla protein (osteocalcin), expressed in osteoblasts and odontoblasts and matrix Gla protein, i.e., expressed in vascular smooth muscle cells and chondrocytes. ${ }^{35}$ Matrix Gla protein acts as an inhibitor of extracellular matrix mineralization and prevents vascular calcification. ${ }^{36}$ Mutations in $M G P$ cause Keutel syndrome, which features chondrodysplasia punctata, midfacial hypoplasia, peripheral pulmonary stenosis and progressive soft tissue, and vascular calcifications. ${ }^{37,38}$ We speculate that ARSE might undergo $\gamma$-carboxylation and have functions similar to matrix Gla protein and osteocalcin, by catalyzing the desulfation of a critical growth plate component that inhibits calcification. Alternatively, ARSE may require vitamin $\mathrm{K}$ for normal growth plate mineralization in a pathway independent of $\gamma$-carboxylation.

In this project, the CETT program fostered collaboration between clinicians, researchers, and clinical laboratories. The 
direct depositing of mutations from the clinical laboratory into a public database ${ }^{39}$ allows access by the entire biomedical community for further investigation.

\section{SUPPLEMENTARY MATERIAL}

Supplementary material is linked to the online version of the paper at http://www.nature.com/gim

\section{ACKNOWLEDGMENTS}

We thank Robert MacKenzie, Rene St-Arnaud, Erminia Di Pietro, and Nicola Brunetti-Pierri for their helpful discussions regarding the ARSE assay. N.B. is funded by the Montreal Children's Hospital Research Institute and C.M.-M. is a graduate student in Human Genetics at McGill University. This study was partially supported by the National Institutes of Health Office of Rare Diseases as part of the pilot Collaboration Education and Test Translation program in CDPX1.

\section{DISCLOSURE}

The authors declare no conflict of interest.

\section{REFERENCES}

1. Sheffield LJ, Danks DM, Mayne V, Hutchinson AL. Chondrodysplasia punctata-23 cases of a mild and relatively common variety. J Pediatr 1976;89:916-923.

2. Curry CJ, Magenis RE, Brown M, et al. Inherited chondrodysplasia punctata due to a deletion of the terminal short arm of an X chromosome. N Engl I Med 1984;311:1010-1015.

3. Nino M, Matos-Miranda C, Maeda M, et al. Clinical and molecular analysis of arylsulfatase $\mathrm{E}$ in patients with brachytelephalangic chondrodysplasia punctata. Am J Med Genet A 2008;146A:997-1008.

4. Wolpoe ME, Braverman N, Lin SY. Severe tracheobronchial stenosis in the $X$-linked recessive form of chondrodysplasia punctata. Arch Otolaryngol Head Neck Surg 2004;130:1423-1426.

5. Franco B, Meroni G, Parenti G, et al. A cluster of sulfatase genes on Xp22.3: mutations in chondrodysplasia punctata (CDPX) and implications for warfarin embryopathy. Cell 1995;81:15-25.

6. Sardiello M, Annunziata I, Roma G, Ballabio A. Sulfatases and sulfatase modifying factors: an exclusive and promiscuous relationship. Hum Mol Genet 2005;14:3203-3217.

7. Cosma MP, Pepe S, Annunziata I, et al. The multiple sulfatase deficiency gene encodes an essential and limiting factor for the activity of sulfatases. Cell 2003;113:445-456.

8. Diez-Roux G, Ballabio A. Sulfatases and human disease. Annu Rev Genomics Hum Genet 2005;6:355-379.

9. Daniele A, Parenti G, d'Addio M, Andria G, Ballabio A, Meroni G. Biochemical characterization of arylsulfatase $E$ and functional analysis of mutations found in patients with X-linked chondrodysplasia punctata. Am J Hum Genet 1998;62:562-572.

10. Brunetti-Pierri N, Andreucci MV, Tuzzi R, et al. X-linked recessive chondrodysplasia punctata: spectrum of arylsulfatase $E$ gene mutations and expanded clinical variability. Am J Med Genet A 2003;117A:164-168.

11. Mandelbrot $L$, Guillaumont $M$, Leclercq $M$, et al. Placental transfer of vitamin $K 1$ and its implications in fetal hemostasis. Thromb Haemost 1988;60:39-43.

12. Brunetti-Pierri N, Hunter JV, Boerkoel CF. Gray matter heterotopias and brachytelephalangic chondrodysplasia punctata: a complication of hyperemesis gravidarum induced vitamin K deficiency? Am J Med Genet A 2007;143:200204.

13. Eash DD, Weaver DD, Brunetti-Pierri N. Cervical spine stenosis and possible vitamin $\mathrm{K}$ deficiency embryopathy in an unusual case of chondrodysplasia punctata and an updated classification system. Am J Med Genet A 2003;122A:70-75.

14. Menger H, Lin AE, Toriello HV, Bernert G, Spranger JW. Vitamin K deficiency embryopathy: a phenocopy of the warfarin embryopathy due to a disorder of embryonic vitamin K metabolism. Am J Med Genet 1997;72:129-134.

15. Herman TE, Lee BC, McAlister WH. Brachytelephalangic chondrodysplasia punctata with marked cervical stenosis and cord compression: report of two cases. Pediatr Radio/ 2002;32:452-456.

16. Jaillet J, Robert-Gnansia E, Till M, Vinciguerra C, Edery P. Biliary lithiasis in early pregnancy and abnormal development of facial and distal limb bones (Binder syndrome): a possible role for vitamin K deficiency. Birth Defects Res Part A Clin Mol Teratol 2005;73:188-193.

17. Chitayat D, Keating S, Zand DJ, et al. Chondrodysplasia punctata associated with maternal autoimmune diseases: expanding the spectrum from systemic lupus erythematosus (SLE) to mixed connective tissue disease (MCTD) and scleroderma report of eight cases. Am J Med Genet A 2008;146A:30383053.

18. Kozlowski K, Basel D, Beighton P. Chondrodysplasia punctata in siblings and maternal lupus erythematosus. Clin Genet 2004;66:545-549.

19. Elçioglu N, Hall CM. Maternal systemic lupus erythematosus and chondrodysplasia punctata in two sibs: phenocopy or coincidence? J Med Genet 1998;35:690-694.

20. Austin-Ward E, Castillo S, Cuchacovich M, et al. Neonatal lupus syndrome: a case with chondrodysplasia punctata and other unusual manifestations. J Med Genet 1998;35:695-697.

21. CETT. Collaboration, Education, and Test Translation (CETT) Program for Rare Genetic Diseases. http://rarediseases.info.nih.gov/cettprogram/default.aspx. Accessed 12 June 2012.

22. PolyPhen-2. http://genetics.bwh.harvard.edu/pph/. Accessed 12 June 2012.

23. SIFT. J. Craig Ventor Institute. http:// sift.jcvi.org/. Accessed 12 June 2012.

24. dbSNP. Short Genetic Variations. http://www.ncbi.n/m.nih.gov/projects/SNP/. Accessed 12 June 2012.

25. Sheffield LJ, Osborn AH, Hutchison WM, et al. Segregation of mutations in arylsulphatase $\mathrm{E}$ and correlation with the clinical presentation of chondrodysplasia punctata. J Med Genet 1998;35:1004-1008.

26. Garnier A, Dauger S, Eurin D, et al. Brachytelephalangic chondrodysplasia punctata with severe spinal cord compression: report of four new cases. Eur $J$ Pediatr 2007; 166:327-331.

27. Casarin A, Rusalen F, Doimo M, et al. X-linked brachytelephalangic chondrodysplasia punctata: a simple trait that is not so simple. Am J Med Genet A 2009;149A:2464-2468.

28. Ghosh D. Mutations in X-linked ichthyosis disrupt the active site structure of estrone/DHEA sulfatase. Biochim Biophys Acta 2004;1739:1-4.

29. Structure of human placental estronedhea sulfatase. http://www.ncbi.nlm.nih. gov/Structure/mmdb/mmdbsrv.cgi?uid=24158. Accessed 12 June 2012.

30. SWISS-MODEL. http:// swissmodel.expasy.org/. Accessed 12 June 2012.

31. Wainwright $\mathrm{H}$, Beighton P. Warfarin embryopathy: fetal manifestations. Virchows Arch 2010;457:735-739.

32. Feteih R, Tassinari MS, Lian JB. Effect of sodium warfarin on vitamin K-dependent proteins and skeletal development in the rat fetus. J Bone Miner Res 1990;5:885-894.

33. Berkner KL. The vitamin K-dependent carboxylase. Annu Rev Nutr 2005;25:127-149.

34. Furie B, Bouchard BA, Furie BC. Vitamin K-dependent biosynthesis of gammacarboxyglutamic acid. Blood 1999;93:1798-1808.

35. Murshed M, Schinke T, McKee MD, Karsenty G. Extracellular matrix mineralization is regulated locally; different roles of two gla-containing proteins. J Cell Biol 2004;165:625-630.

36. Danziger J. Vitamin K-dependent proteins, warfarin, and vascular calcification. Clin J Am Soc Nephro/ 2008;3:1504-1510.

37. Munroe PB, Olgunturk RO, Fryns JP, et al. Mutations in the gene encoding the human matrix Gla protein cause Keutel syndrome. Nat Genet 1999;21:142144.

38. Meier M, Weng LP, Alexandrakis E, Rüschoff J, Goeckenjan G. Tracheobronchial stenosis in Keutel syndrome. Eur Respir J 2001;17:566-569.

39. X-chromosome gene database, ARSE gene. https://grenada.lumc.nI/LOVD2/ MR/home.php?select_db=ARSE. Accessed 12 June 2012. 